\title{
Autoantibodies against the Amino-terminal Cadherin-like Binding Domain of Pemphigus Vulgaris Antigen Are Pathogenic
}

Masayuki Amagai, Sarolta Karpati, Ronald Prussick, Vera Klaus-Kovtun, and John R. Stanley

Dermatology Branch, National Cancer Institute, National Institutes of Health, Bethesda, Maryland 20892

\begin{abstract}
Complementary DNA cloning of the $130-\mathrm{kD}$ pemphigus vulgaris (PV) autoantigen (PVA) has indicated that it is a member of the cadherin family of $\mathrm{Ca}^{2+}$-dependent cell adhesion molecules. By homology with typical cadherins, PVA has five extracellular domains (EC1 through EC5). To localize immunogenic domains and to determine whether antibodies against them might be pathogenic, we produced $\beta$-galactosidase fusion proteins with cDNA encoding different portions of the extracellular domains of PVA (EC1-2, EC3-5, and each individual domain). Immunoblot analysis of these fusion proteins with 23 PV patients' sera demonstrated that major immunogenic regions of PVA are located on the EC1, EC2, and EC4 domains. IgG was affinity-purified from $P V$ sera on fusion proteins representing the amino (EC1-2) and carboxy (EC3-5) terminus of the extracellular PVA, and injected into neonatal mice. PV IgG affinity-purified on the EC1-2 fusion protein caused suprabasilar acantholysis, the typical histological finding of PV, but IgG affinity-purified on the EC3-5 fusion protein or $\beta$-galactosidase alone did not. These results indicate that at least one pathogenic epitope, which is sufficient to cause suprabasilar acantholysis in neonatal mice, is located on the amino-terminal region of PVA, an area thought to be important in cadherin homophilic adhesion. (J. Clin. Invest. 1992. 90:919-926.) Key words: acantholysis $\bullet$ autoimmunity $\bullet$ cell adhesion $\bullet$ desmosome $\bullet$ immunogenic domain
\end{abstract}

\section{Introduction}

Pemphigus vulgaris (PV) ${ }^{1}$ is a life-threatening autoimmune blistering disease of skin and mucous membranes. Autoantibodies from patients with PV bind the keratinocyte cell surface and cause loss of cell adhesion with resultant blister formation (1). PV antigen (PVA), which is defined by autoantibodies from these patients, has been characterized by immunoprecipitation and immunoblotting as a $130-\mathrm{kD}$ glycoprotein (2-5). Recently, by immunoscreening with affinity-purified PV IgG, we have isolated cDNA clones encoding PVA from an expres-

Address reprint requests to Dr. Amagai, Dermatology Branch, Building 10, Room 12N238, National Cancer Institute, National Institutes of Health, Bethesda, MD 20892.

Received for publication 10 March 1992.

1. Abbreviations used in this paper: DGI, desmoglein I; FP, fusion protein; $\beta$-Gal, cro- $\beta$-galactosidase; IF, immunofluorescence; NHEK, cultured normal human epidermal keratinocytes; PCR, polymerase chain reaction; $\mathrm{PF}$, pemphigus foliaceus; $\mathrm{PV}$, pemphigus vulgaris; PVA, the $130-k D$ pemphigus vulgaris antigen; TBS, Tris-buffered saline.

The Journal of Clinical Investigation, Inc.

Volume 90, September 1992, 919-926 sion library reflecting mRNA sequences from normal human keratinocytes (6). The deduced amino acid sequence of PVA was unique, but showed marked homology with members of the cadherin family of $\mathrm{Ca}^{2+}$-dependent cell adhesion molecules.

Cadherins mediate homophilic binding and are thought to be important in establishing and maintaining epithelial and neural tissue integrity $(7,8)$. The originally described cadherins include E-cadherin (also called uvomorulin) $(9,10), \mathrm{N}$ cadherin (11-13), P-cadherin (14, 15), and liver cell adhesion molecule (16), which are all linked to the actin filament network and can be found in the cell-cell adherens-type junction (17). More recently described members of the cadherin superfamily include desmoglein I (DGI) (18-21 ) and desmocollins I and II $(22,23)$, glycoproteins of the desmosome type of cell adhesion junction which is linked to intermediate filaments (17).

PVA is similar in overall structure to these other cadherins (6). Like other cadherins, PVA has five extracellular domains of approximately equal size, termed EC1 (amino terminus) through EC5 (carboxy terminus of the extracellular portion), which, except for EC5, have homology with each other. As for typical cadherins, in PVA the homology is greatest among EC1, EC2, and EC3. The homology of the EC1 through EC4 domains of PVA to each corresponding domain of the originally described cadherins is $\sim 50 \%$. However, for PVA domains EC1, EC2, and EC3, the homology to the corresponding domains in DGI, 75-80\%, is much greater than to the corresponding domains in the original cadherins.

Studies using chimeric cadherin molecules between E- and P-cadherin, amino acid substitutions by site-directed mutagenesis, and epitope mapping of monoclonal antibodies that inhibit homophilic binding, have shown that the 113 amino-terminal residues (EC1 and part of the EC2 domain) are important for determining binding function and specificity of binding (24). The highly conserved amino acid sequence histidine-alanine-valine (H-A-V) of typical cadherins (7), which is present in EC1 and thought to be the center of homophilic binding (25), is represented in PVA and DGI by the conservatively substituted sequence arginine-alanine-leucine (R-A-L) $(6,18)$. Furthermore, the homology of PVA with cadherins in these amino-terminal domains is the same across species lines, suggesting that, inasmuch as these areas have been evolutionarily conserved, they are functionally important. These observations suggest that the amino-terminal domains of PVA, like those of the original cadherins, might be important for the binding function of PVA, and thereby contribute to the structural integrity of the epidermis.

Therefore, in this study, we wanted to determine whether (a) the amino terminus of PVA is immunogenic in PV patients and $(b)$ autoantibodies against this portion of the molecule can induce the loss of cell adhesion in a neonatal mouse model of disease. 


\section{Methods}

Human sera. Sera from patients with clinically and histologically typical PV showed characteristic cell surface immunofluorescence on monkey esophagus and immunoprecipitated or immunoblotted the 130-kD PVA $(1,5)$. PV sera 1238 and 1491 were obtained by plasmapheresis. Disease control sera were obtained from patients with clinically and histologically typical pemphigus foliaceus (PF). PF is an autoimmune blistering disease of epidermis, in which autoantibodies bind DGI (26-28). These PF control sera showed characteristic immunofluorescence findings. Normal human sera were also used as controls.

Immunofluorescence. Indirect immunofluorescence with PV sera, affinity-purified PV IgG, or sera from neonatal mice was performed on monkey esophagus, normal human epidermis or neonatal mouse epidermis as previously described (29).

Production of PVA fusion proteins. Seven fusion proteins(FPs) that represent different portions of the extracellular domains of PVA (EC12 FP, EC3-5 FP, EC1 FP, EC2 FP, EC3 FP, EC4 FP, and EC5 FP) were produced (Fig. 1). EC3-5 FP was referred to as MJ315 FP in a previous paper (6). To obtain the other FPs, we used a full-length (for the coding region) form of PVA cDNA as a substrate for polymerase chain reaction (PCR) amplification. To obtain full-length PV cDNA, the extended clones of PVA cDNA, E12 and E33 (6), were cut out from plasmids by double digestion with $\mathrm{XhoI}$ and $\mathrm{XbaI}$, and $\mathrm{XbaI}$ and NotI, respectively. The digested E12 and E33 were ligated at their common Xbal site and subcloned into pBluescript (Strategene, La Jolla, CA), at $\mathrm{Xhol}$ and NotI sites, to yield pBlue-PV which contained the full-length form of PVA cDNA. cDNA inserts representing each domain were excised from pBlue-PV by PCR amplification with primers that contained the appropriate restriction sites for directional, and in frame, subcloning to the pUEX1 expression plasmid vector (Amersham Corp., Arlington Heights, IL) (Table I). pUEX1 produces an FP with cro- $\beta$-galactosidase $(\beta$-Gal). The sequences around the cloning sites were confirmed by DNA sequencing.

Transformants of these expression vectors were grown at $30^{\circ} \mathrm{C}$ in Luria-Bertani medium; then the expression of FPs was induced by transferring the host cells to $42^{\circ} \mathrm{C}$ for $2 \mathrm{~h}$. Host cell pellets were then suspended in water and stored at $-70^{\circ} \mathrm{C}$ until use.

The correct size FPs were produced by each pUEX1 subclone, as confirmed by immunoblotting with anti- $\beta$-galactosidase antibodies (Tago, Inc., Burlingame, CA) and PV sera.

Immunoblotting. E. coli suspensions or proteins from cultured normal human epidermal keratinocytes (NHEK) were extracted with SDS sample buffer with reduction, separated by SDS-PAGE, and transferred to nitrocellulose membranes $(5,6,30)$. Immunoblotting with a

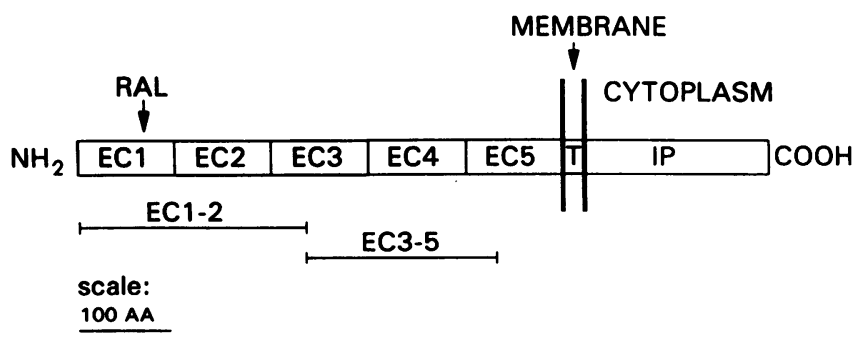

Figure 1. Location of FPs representing different portions of the extracellular domains of PVA. Seven FPs were produced, the amino(EC1-2) and carboxy- (EC3-5) portions of the extracellular domain of PVA and each individual domain (EC1, EC2, EC3, EC4, and EC5). RAL indicates the portion of the arginine-alanine-leucine sequence, which is the same region as, and homologous to, the conserved histidine-alanine-valine sequence of the originally described cadherins. These sequences are hypothesized to be important in cell adhesion. $T$ and $I P$, transmembrane portion and intracytoplasmic regions, respectively.
Table I. Summary of PVA FP

\begin{tabular}{|c|c|c|c|c|}
\hline \multirow[b]{3}{*}{ FP } & \multirow{2}{*}{\multicolumn{2}{|c|}{ Cloning site }} & \multicolumn{2}{|c|}{ Amino acid sequency of PVA part } \\
\hline & & & & \\
\hline & $5^{\prime}$ & $3^{\prime}$ & $\begin{array}{l}\text { Amino } \\
\text { terminus }\end{array}$ & $\begin{array}{l}\text { Carboxy } \\
\text { terminus }\end{array}$ \\
\hline
\end{tabular}

$k D$

$\begin{array}{lllrll}\text { EC1-2 } & \text { SmaI } & \text { BamHI } & \text { REWVKFAK--- } & --- \text { TSGNEGNW } & 146 \\ \text { EC3-5 } & \text { BamHI } & \text { PstI } & \text { EGNWFEIQ--- } & --- \text { TLNNRYTG } & 139 \\ \text { EC1 } & \text { SmaI } & \text { BamHI } & \text { EWVKFAKP--- } & --- \text { INDNPPVF } & 128 \\ \text { EC2 } & \text { BamHI } & \text { PstI } & \text { SQQIFMGE--- } & --- \text { VNDNFPMF } & 128 \\ \text { EC3 } & \text { BamHI } & \text { PstI } & \text { RDSQYSAR--- } & --- \text { NVREGIAF } & 130 \\ \text { EC4 } & \text { BamHI } & \text { PstI } & \text { RPASKTFT--- } & -- \text { PDFNDNCP } & 128 \\ \text { EC5 } & \text { BamHI } & \text { PstI } & \text { TAVLEKDA--- } & --- \text { YGRPHSGR } & 129\end{array}$

* Total molecular mass $\left(M_{\mathrm{r}}\right)$ of FP, which includes $116 \mathrm{kD}$ of $\beta-\mathrm{Gal}$ contributed by pUEX1 vector.

first antibody at $4^{\circ} \mathrm{C}$ with overnight incubation was performed with 1:50 or 1:100 dilutions of various human sera, as well as the bound or unbound fraction of human sera passed over affinity columns. Rabbit anti- $\beta$-galactosidase antibody ( Tago, Inc.) was also used as a first antibody to detect the FP. Alkaline phosphatase-conjugated goat anti-human IgG (Zymed Laboratories, Inc., San Francisco, CA) or goat antirabbit IgG (Tago, Inc.) was used as a second antibody and incubated at room temperature for $1 \mathrm{~h} \mathrm{(6)}$.

Affinity purification of $P V I g G$. The $E$. coli suspension obtained after induction of FP synthesis was incubated with $1 / 9$ vol of $0.5 \mathrm{M}$ Tris- $\mathrm{HCl}, \mathrm{pH} 7.5,1.5 \mathrm{M} \mathrm{NaCl}$ and $1 / 20$ vol of $10 \mathrm{mg} / \mathrm{ml}$ lysozyme at $37^{\circ} \mathrm{C}$ for $15 \mathrm{~min}$, sonicated for $20 \mathrm{~s}$ on ice, and then centrifuged for 10 min at $2,400 \mathrm{~g}$. The precipitated FP was partially purified by washing sequentially with $0.5 \%$ Triton X-100 in $150 \mathrm{mM} \mathrm{NaCl}, 10 \mathrm{mM}$ EDTA, $10 \mathrm{mM}$ Tris- $\mathrm{HCl}, \mathrm{pH} 7.5$, then with $2 \mathrm{M}$ urea in $100 \mathrm{mM}$ Tris- $\mathrm{HCl}, \mathrm{pH}$ 8. The remaining precipitated FP was dissolved in $8 \mathrm{M}$ urea, $100 \mathrm{mM}$ Hepes, pH 7.3 and dialyzed against $50 \mathrm{mM}$ Hepes, pH 7.3 overnight.

Approximately $5 \mathrm{mg}$ of purified FP was coupled to $3-5 \mathrm{ml}$ of washed Affi-Gel 10 beads (Bio-Rad Laboratories, Richmond, CA) in $50 \mathrm{mM}$ Hepes, pH 7.3, by mixing overnight at $4^{\circ} \mathrm{C}$. The gel slurries were loaded into mini-columns (Bio-Rad Laboratories) and washed

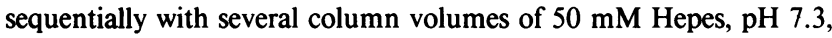
followed by Tris-buffered saline (TBS): $150 \mathrm{mM} \mathrm{NaCl}, 10 \mathrm{mM}$ Tris$\mathrm{HCl}, \mathrm{pH}$ 7.5. The coupling of FPs to Affi-Gel 10 was confirmed by the disappearance on SDS-PAGE of the FP bands. $10-50 \mathrm{ml}$ of PV sera diluted three times with TBS was run over each column with continuous recirculation for $12-48 \mathrm{~h}$. Sera that passed through the column were collected as the pass-through fraction. The columns were washed extensively with TBS to remove unbound components. Bound antibodies were eluted from columns with $50 \mathrm{mM}$ glycine, $500 \mathrm{mM} \mathrm{NaCl}, \mathrm{pH}$ 2.3 , immediately neutralized with $1 / 5$ vol of $2 \mathrm{M}$ Tris- $\mathrm{HCl} \mathrm{pH} \mathrm{7.5,} \mathrm{and}$ dialyzed against PBS pH 7.4, at $4^{\circ}$ overnight. The quality of eluted affinity-purified antibodies, as well as sera depleted of FP reactivity, were checked by indirect immunofluorescence (IF) and immunoblotting against NHEK extracts. Finally, eluted antibodies were concentrated down from $\sim 15 \mathrm{ml}$ to $150-300 \mu$ l with microconcentrators (Centricon 30, Amicon, Beverly, MA) to inject into neonatal mice. From the pass-through fraction of PV sera, IgG was prepared by precipitation with $40 \%$ ammonium sulfate, dialyzed against $P B S$, and concentrated to $\sim 100 \mathrm{mg} / \mathrm{ml}$.

Neonatal mouse model of $P V$. Concentrated antibodies from the affinity-purified or pass-through fractions from PV sera were injected subcutaneously into neonatal BALB /c mice ( $<24 \mathrm{~h}$ of age) through a 30 -gauge needle as described previously $(31,32)$. Neonates were examined and biopsied $18 \mathrm{~h}$ after injection. Serum was obtained from mice at this time and assayed for human PV antibodies by indirect IF. Skin was studied by light microscopy for evaluation of histological changes 
and by direct IF for detection of in vivo bound PV antibodies on the cell surface.

\section{Results}

Major immunogenic regions of PVA are on extracellular domains $E C 1, E C 2$, and EC4. To determine the immunogenicity of the extracellular domains of PVA, we produced seven $\beta$-Gal FPs that represent different regions of the extracellular domains of PVA (Fig. 1 and Table I). EC1-2 FP and EC3-5 FP represent amino- and carboxy-terminal portions, respectively, of extracellular PVA. FPs representing each extracellular domain, EC1-EC5, were also produced. These FPs, as well as extracts of NHEK cells cultured in high $\mathrm{Ca}^{2+}$ to induce increased PVA synthesis (6), were subjected to immunoblot analysis with 23 PV sera, 15 PF sera, and 5 normal sera. To exclude the possibility that some of these sera had antibodies against $\beta$-Gal core protein, which would give pseudopositivity, $\beta$-Gal, produced by the pUEX1 expression vector without an insert, was always applied to gels with these FPs. None of the sera we tested reacted strongly with $\beta$-Gal by immunoblot. The position of the FPs and $\beta$-Gal on these immunoblots was confirmed by staining with rabbit anti- $\beta$-Gal antibodies.

Table II summarizes the results of immunoblot analysis and Fig. 2 shows representative immunoblots. 12 of 23 PV sera reacted with EC1-2 FP, and out of these 12 sera 11 and 10 sera recognized EC1 FP and EC2 FP, respectively. EC2 FP was also recognized by one additional PV sera that did not bind to EC12. EC3-5 FP was bound by 6 PV sera out of the 23, and 5 of these 6 sera also bound to the EC4 FP. The EC4 FP was recognized by an additional three sera which did not bind to the EC3-5 FP. The EC3 FP and EC5 FP were recognized by only one serum each. All sera that recognized any of these FPs also bound the 130-kD PVA from extracts of NHEK. Moreover, four sera that bound the 130-kD PVA from NHEK extracts did not recognize any of these FPs. Two sera did not bind the PVA from NHEK extract and did not recognize any of these FPs. None of 15 PF sera nor 5 normal control sera recognized any of these FPs.

These results indicate that major immunogenic regions of PVA are on the EC1, EC2, and EC4 domains, as determined by this type of immunoblot assay.

Affinity purification of $P V \operatorname{IgG}$. Because the amino-terminus of cadherins is thought to be important for homophilic binding interactions, we attempted to determine whether PV antibodies directed against this region, as opposed to the carboxy-terminus of the extracellular PVA, are pathogenic. PV IgG was affinity-purified on EC1-2 and EC3-5 FP columns from two PV sera ( 1238 and 1491). These PV sera were chosen because we had large quantities obtained by plasmapheresis. A $\beta$-Gal affinity column was used as a control to eliminate the possibility of nonspecific binding of PV IgG to these affinity columns. Affinity-purified IgG from $10-50 \mathrm{ml}$ of PV sera was examined by indirect IF and immunoblotting. Antibodies af-

Table II. Immunoblot analysis of PVA FPs with PV Patients' Sera

\begin{tabular}{|c|c|c|c|c|c|c|c|c|c|c|}
\hline No. & PV sera & ECl-2 & EC3-5 & $\mathrm{ECl}$ & EC2 & EC3 & EC4 & EC5 & NHEK ${ }^{*}$ & IIF $^{\ddagger}$ \\
\hline 1 & 1338 & $++^{\S}$ & + & ++ & + & - & + & - & ++ & 1280 \\
\hline 2 & 1181 & + & ++ & + & + & - & ++ & - & + & 80 \\
\hline 3 & 1405 & ++ & - & + & + & - & + & - & + & 640 \\
\hline 4 & 1213 & ++ & - & + & + & + & + & - & + & $>320$ \\
\hline 5 & 1406 & ++ & - & ++ & + & - & - & - & ++ & 40 \\
\hline 6 & 443 & ++ & - & ++ & + & - & - & - & ++ & 160 \\
\hline 7 & 305 & ++ & - & ++ & + & - & - & - & ++ & 320 \\
\hline 8 & 1404 & + & - & ++ & + & - & + & - & ++ & 160 \\
\hline 9 & 1407 & + & - & + & + & - & - & - & ++ & 160 \\
\hline 10 & 709 & ++ & - & ++ & - & - & - & + & ++ & $>320$ \\
\hline 11 & 820 & ++ & - & ++ & - & - & - & - & ++ & 160 \\
\hline 12 & 1408 & + & - & - & ++ & - & - & - & + & 160 \\
\hline 13 & 1238 & - & ++ & - & - & - & ++ & - & ++ & $>320$ \\
\hline 14 & 951 & - & ++ & - & - & - & + & - & ++ & 80 \\
\hline 15 & 972 & - & + & - & - & - & ++ & - & + & 160 \\
\hline 16 & 1364 & - & + & - & - & - & - & - & + & $>320$ \\
\hline 17 & 633 & - & - & - & + & - & - & - & + & 160 \\
\hline 18 & 1491 & - & - & - & - & - & - & - & + & $>160$ \\
\hline 19 & 1042 & - & - & - & - & - & - & - & + & 160 \\
\hline 20 & 1090 & - & - & - & - & - & - & - & ++ & 640 \\
\hline 21 & 813 & - & - & - & - & - & - & - & + & 1280 \\
\hline 22 & 913 & - & - & - & - & - & - & - & - & 80 \\
\hline 23 & 1403 & - & - & - & - & - & - & - & - & 640 \\
\hline Total & & 12 & 6 & 11 & 11 & 1 & 8 & 1 & 21 & \\
\hline
\end{tabular}

* NHEK indicates immunoblot results on extracts of NHEK cultured in high $\mathrm{Ca}^{2+}$ medium; + denotes positive staining of the $130-\mathrm{kD}$ band. ${ }^{\ddagger}$ IIF indicates indirect immunofluorescence titer of sera on monkey esophagus. ${ }^{\$}++,+$, and - indicate strongly positive, positive, and negative staining, respectively. 


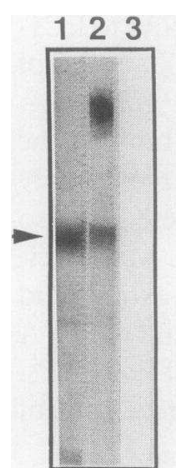

NHEK

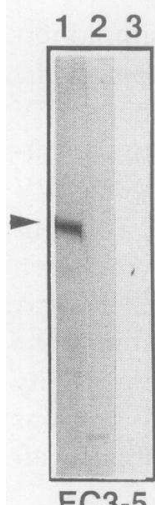

EC3-5

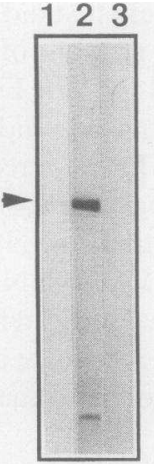

EC1-2

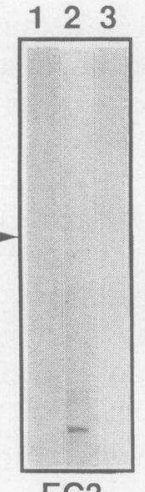

EC3

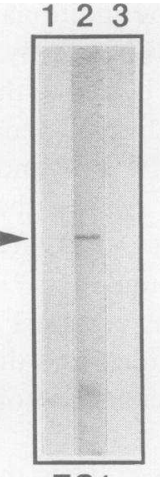

EC1

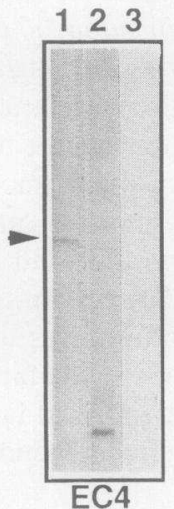

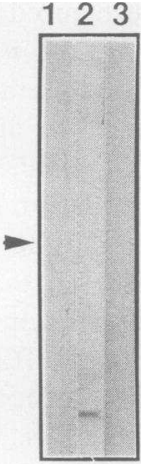

EC2

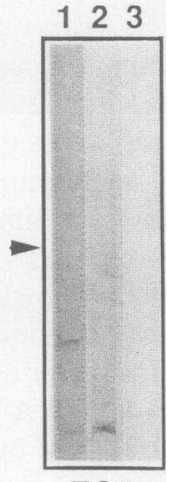

EC5
Figure 2. Immunoblot analysis of PVA and PVA FPs with PV and PF sera. NHEK extract and the seven FPs were incubated with two PV sera (lane 1, serum 1238; lane 2, serum 820) and one PF serum (lane $3)$. Arrowheads indicate the positions of the 130-kD PVA from NHEK extracts and each individual FP. The positions of FPs were confirmed by anti- $\beta$-Gal antibodies (not shown).

finity-purified on both EC1-2 and EC3-5 FPs stained the cell surface of monkey esophagus, normal human epidermis, and neonatal mouse skin in the same pattern as do PV sera, but with much less background (Fig. 3, $B-G$ ). The titers of concentrated IgG affinity-purified on EC1-2 and EC3-5 FPs were both $\sim 640$ on normal human skin, 320 on monkey esophagus, and 320 on neonatal mouse skin. Both of these affinity-purified IgGs recognized the $130-\mathrm{kD}$ PVA on immunoblot (Fig. 4, lanes 7 and 8 ). IgG that nonspecifically bound $\beta$-Gal did not show any reactivity to the keratinocyte cell surface by immunofluorescence (Fig. $3 A$ ) nor the 130-kD PVA by immunoblot (Fig. 4, lane 9). IgG affinity-purified on EC3-5 FP from PV serum 1238 recognized EC3-5 FP (Fig. 4, lane 2), but IgG affinity-purified on EC1-2 FP or $\beta$-Gal did not (Fig. 4, lanes 1 and 3 ), indicating the specificity of these affinity-purified IgGs. The pass-through IgG from the EC3-5 FP column, but not from the EC1-2 FP or $\beta$-Gal column, showed depletion of the reactivity against the EC3-5 FP (Fig. 4, lanes 4-6). The passthrough IgG from the EC1-2 and EC3-5 FP columns had diminished reactivity against the $130-\mathrm{kD}$ PVA on immunoblot, compared with the pass-through IgG from the $\beta$-Gal column (Fig. 4, lanes 10-12).

These high-titer, affinity-purified IgGs provided reagents to examine the pathogenicity of antibodies directed against the EC1-2 and EC3-5 regions of PVA.
$P V I g G$ directed against the amino terminus of the PVA is pathogenic in neonatal mice. PV IgG affinity-purified on EC12 , EC3-5, and $\beta$-Gal FPs were injected subcutaneously into BALB/c neonatal mice which were examined $18 \mathrm{~h}$ after injection (Table III). IgG affinity-purified on EC1-2 from both PV sera 1238 and 1491 caused suprabasilar acantholysis, the characteristic histological finding of PV (Fig. 5, $A$ and $B$ ). This suprabasilar acantholysis was observed in all the mice injected with IgG affinity-purified on ECl-2. In addition, the skin of these mice showed human IgG deposition on the cell surface of epidermis by direct IF (Fig. 5, $B$ and $C$ ), and the titer of human PV IgG in the sera of these mice was $20-80$, as determined by indirect IF on monkey esophagus. However, gross blister formation was not observed in any of these mice. In contrast to IgG affinity-purified on EC1-2, PV IgG affinity-purified on EC3-5 failed to induce acantholysis in any of the mice injected, even though the titers of human PV IgG in these mice sera were equivalent to those of mice injected with IgG affinity-purified on EC1-2 (Table III). However, in spite of similar serum titers of PV IgG, direct IF in the skin of mice injected with IgG affinity-purified on EC3-5 was negative or weaker than that of mice injected with IgG affinity-purified on EC1-2 (Fig. 5, C and $D$ ). Mice injected with IgG bound to the $\beta$-Gal column showed negative direct IF and negative indirect IF and did not show acantholysis ( Table III).

These observations indicate that ECl-2 contains at least one pathogenic epitope which is sufficient to cause suprabasilar acantholysis in neonatal mice and provide the first direct evidence that the same antibodies that bind PVA are pathogenic in PV.

To determine whether the epitope(s) that we identified on EC1-2 is (are) the only pathogenic epitope(s) on PVA, we injected neonatal mice with IgG from PV sera that were depleted of EC1-2 FP reactivity by passing them over an EC1-2 FP column. The pass-through IgG from this EC1-2 FP column did not bind EC1-2 FP on a second column, as shown by negative indirect IF of the eluted IgG from this second column. This EC1-2 pass-through IgG, depleted of EC1-2 FP reactivity, was able to induce not only histological suprabasilar acantholysis, but also gross blisters, in these mice. This observation indicates that the EC1-2 FP column could only absorb out a small portion of the pathogenic antibodies from PV sera and that PVA has other pathogenic epitope(s), which, most likely, are conformational epitopes that were not generated by this bacterial FP system.

\section{Discussion}

In this study, we aimed to determine whether some regions of PVA are more immunogenic than others and to study the pathogenicity of PV antibodies against specific domains. The homology of PVA with cadherins led us to hypothesize that the amino terminus of the extracellular PVA might be important for keratinocyte cell-cell adhesion. We further hypothesized that patients' autoantibodies against PVA might directly interfere with this binding region and cause loss of cell adhesion and resultant blister formation. Therefore, we first determined whether PV IgG showed preferential binding to the amino terminal region of PVA, and then determined whether antibodies 

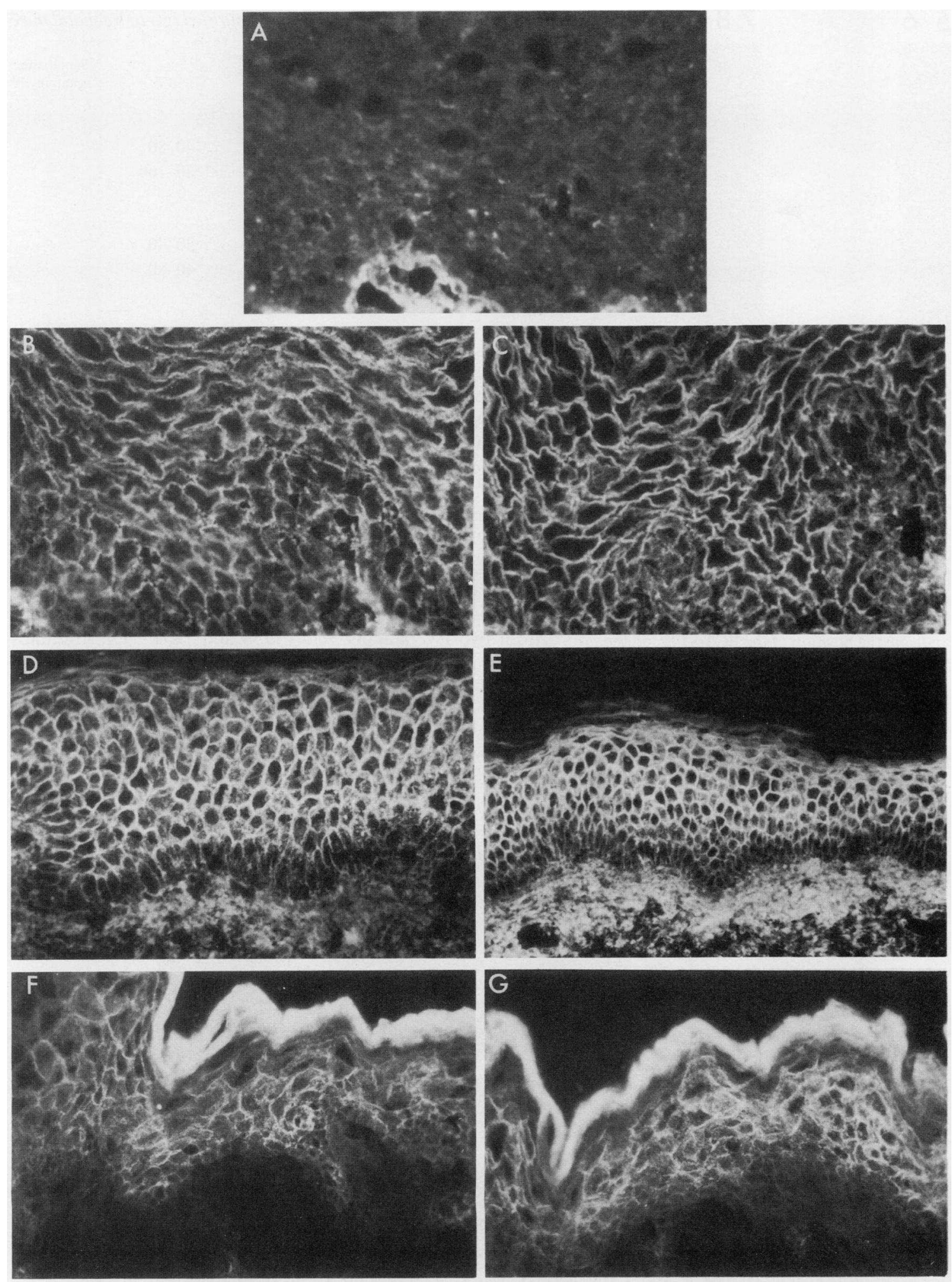

Figure 3. Indirect IF of IgG affinity-purified on EC1-2 FP, EC3-5 FP, and $\beta$-Gal columns. $(A-C)$ Monkey esophagus, $(D$ and $E)$ normal human skin, and $(F$ and $G)$ normal neonatal mouse skin were incubated with $\operatorname{IgG}$ affinity-purified on the $\operatorname{EC1-2~} \mathrm{FP}(B, D$, and $F)$, the EC3-5 FP $(C$, $E$, and $G)$, and $\beta$-Gal $(A) . \times 100$.

against this region were pathogenic in a well-established neonatal mouse model of disease.

Seven different FPs, which represented different portions of PVA, were produced. About half of the sera tested bound EC1-
2 FP, and most of them also reacted with EC1 FP and EC2 FP. A quarter of the sera reacted with EC3-5 FP, and most of them reacted with EC4 FP, but not with EC3 or EC5 FP. These findings indicate that there are major immunogenic regions of 


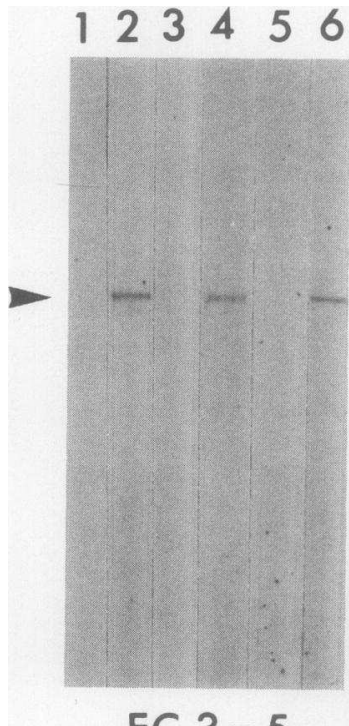

EC $3-5$
789101112

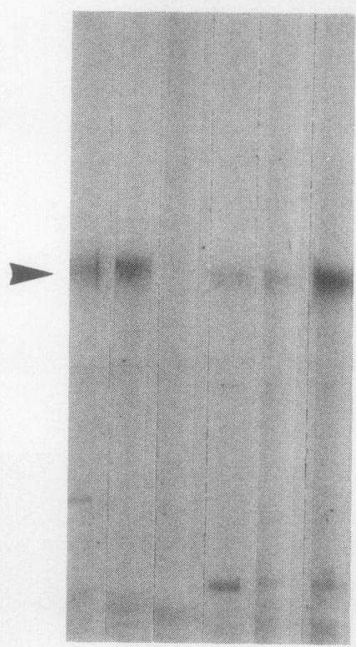

NHEK

Figure 4. Immunoblot analysis of affinity-purified IgG and passthrough IgG of PV serum 1238 from EC1-2 FP, EC3-5 FP, and $\beta$-Gal columns. EC3-5 FP (left panel) and NHEK extract (right panel) were incubated with IgG affinity-purified on EC1-2 (lanes 1 and 7), EC3-5 (lanes 2 and 8 ), $\beta$-Gal (lanes 3 and 9 ) and pass-through IgG from EC1-2 (lanes 4 and 10), EC3-5 (lanes 5 and 11 ), and $\beta$-Gal (lanes 6 and 12). Arrowheads indicate the position of the EC3-5 FP and the 130-kD PVA, respectively.

Table III. Passive transfer of Affinity-purified IgG to Neonatal mice

\begin{tabular}{lcccc}
\hline $\begin{array}{c}\text { Antibodies } \\
\text { injected }\end{array}$ & $\begin{array}{c}\text { Number } \\
\text { of mice }\end{array}$ & DIF* & IIF & $\begin{array}{c}\text { Suprabasilar } \\
\text { acantholysis }\end{array}$ \\
\hline PV serum 1238 & & & & \\
EC1-2 IgG & 3 & ++ & $40-80$ & + \\
EC3-5 IgG & 5 & - & $80-160$ & - \\
$\beta-$-Gal IgG & 4 & - & - & - \\
PV serum 1491 & & & & + \\
EC1-2 IgG & 2 & ++ & $20-40$ & + \\
EC3-5 IgG & 2 & + & $40-80$ & - \\
$\beta-$-Gal IgG & ND & & & \\
\hline
\end{tabular}

* Direct IF on neonatal mouse skin with FITC-conjugated antihuman IgG antibodies. ${ }^{\ddagger}$ Indirect IF titer on monkey esophagus with mice sera at the time of biopsy ( $18 \mathrm{~h}$ after injection). ${ }^{\S}+$ indicates suprabasilar acantholysis was observed by light microscopy. ND, not determined.

PVA on the amino-terminal EC1 and EC2 domains. However, some PV sera contained antibodies that bound the more carboxy-terminal extracellular domain (EC4).

To characterize potentially pathogenic domains of PVA, IgG was affinity-purified using EC1-2 and EC3-5 FP columns from two PV patients' sera (1238 and 1491). On immunoblots, serum 1238 recognized the EC3-5 FP but not the EC1-2
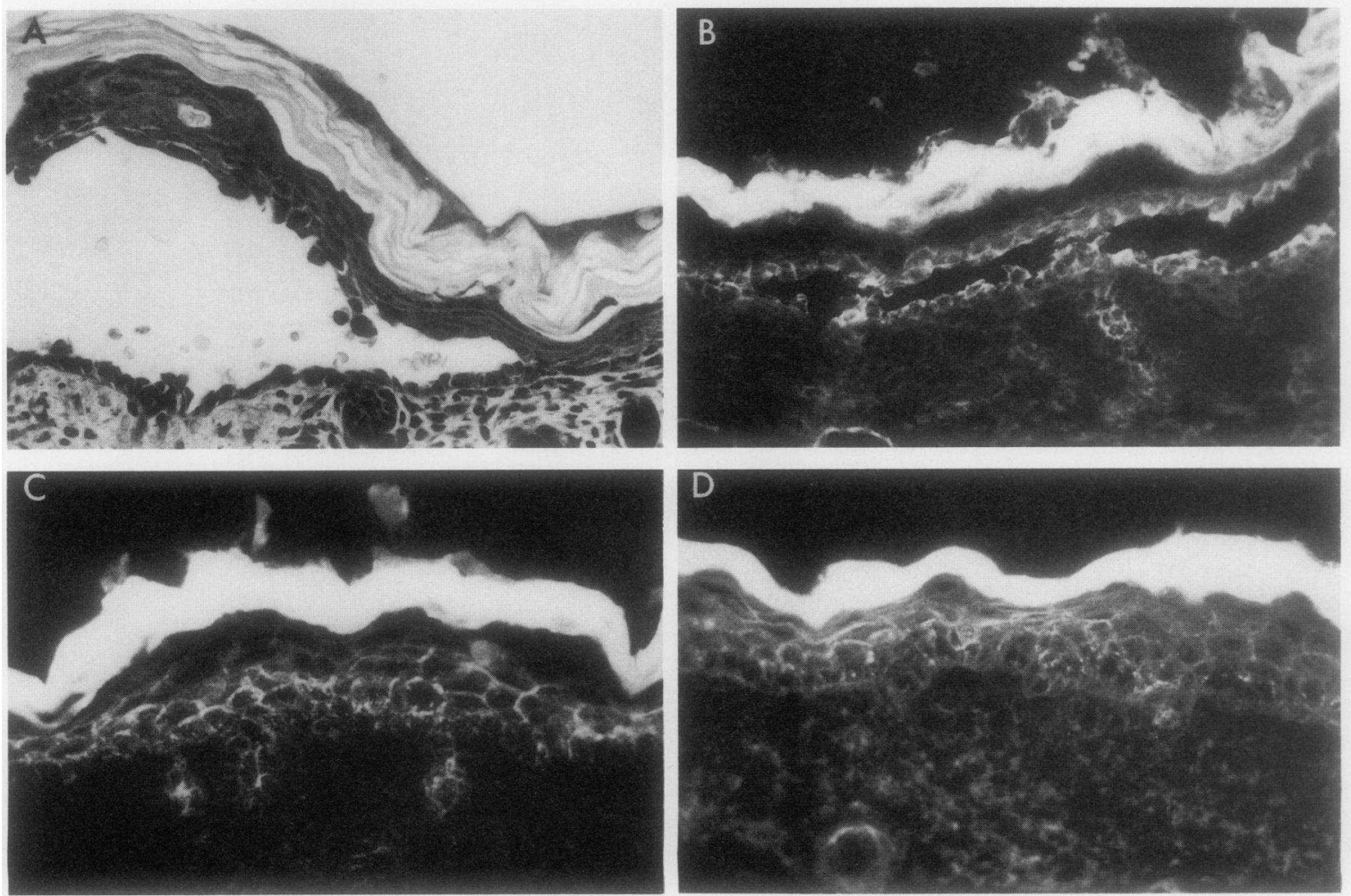

Figure 5. Histology and direct IF of mice injected with IgG affinity-purified on EC1-2 and EC3-5 FPs. $(A)$ Histology and $(B)$ direct IF of the mice injected with IgG affinity-purified on EC1-2 show suprabasilar acantholysis and cell surface IF staining, both typical of PV. The staining intensity of the direct IF on epidermis from the mice injected with IgG affinity-purified on EC1-2 $(C)$ is stronger than that of the mice injected with IgG affinity-purified on EC3-5 (D). 
FP, whereas serum 1491 did not bind either FP. Nevertheless, nondenatured EC1-2 and EC3-5 FPs were able to bind and affinity-purify IgG from both of these sera. These affinity-purified IgGs bound the cell surface of monkey esophagus, human epidermis and neonatal mouse epidermis by indirect IF (Fig. 3, $B-G$ ) and recognized the $130-k D$ PVA by immunoblot (Fig. 4, lanes 7 and 8 ). Control experiments showed that eluted IgG from $\beta$-Gal affinity columns was negative by indirect IF (Fig. 3 $A$ ) and immunoblot (Fig. 4, lane 9). These findings suggest that nondenatured FPs may also express some native epitopes which are destroyed on these FPs by the denaturing conditions of immunoblotting.

In the neonatal mouse model of $\mathrm{PV}, \mathrm{IgG}$ affinity-purified on EC1-2 could cause suprabasilar acantholysis in the epidermis, the characteristic histological finding of PV, but IgG affinity-purified on EC3-5, even though of equivalent IF titer, could not. Therefore by homology with original cadherins, we speculate that antibodies against the amino-terminal domains of PVA might directly interfere with the binding domain of PVA and initiate suprabasilar acantholysis.

In neonatal mice injected with IgG affinity-purified on EC3-5, direct IF was weaker than that of the mice injected with IgG affinity-purified on EC1-2. However the indirect IF titers of sera from the mice injected with EC3-5 IgG were equivalent to those of the mice injected with EC1-2 IgG, when tested on both monkey esophagus and neonatal mouse skin. These observations suggest that epitopes expressed on the EC3-5 FP are somewhat hidden or masked in vivo.

It is potentially significant that the IgG affinity-purified on EC1-2 caused only microscopic blisters, but did not cause gross blisters. This finding was probably not due simply to low amounts of injected PV IgG because the indirect IF titer of sera from mice injected with IgG affinity-purified on EC1-2 was $20-80$, which is high enough to cause gross blisters $(31,32)$. Furthermore, in some experiments, the IgG affinity-purified on EC1-2 was prepared from as much as $50 \mathrm{ml}$ of PV sera, concentrated down to $150 \mu \mathrm{l}$, and injected into one mouse. That antibodies against epitopes other than expressed on the EC1-2 FP are pathogenic was shown by using PV IgG depleted of reactivity against the EC1-2 FP. This IgG caused gross blisters in neonatal mice. We speculate that, although antibodies affinity-purified against the amino-terminal domain of PVA can clearly initiate blister formation, antibodies against additional epitopes might augment blister formation, perhaps through the induction of proteinase release (33) or complement activation $(34,35)$, which may be necessary to destroy additional adhesion molecules on keratinocytes.

The findings of this study provide the first direct evidence that the $130-\mathrm{kD}$ PVA is a pathogenic autoantigen of PV and indicate that the autoantibodies against the amino-terminal cadherin-like binding domain of PVA are sufficient to initiate suprabasilar acantholysis. Identifying other pathogenic epitopes should help to further understand, not only the function of PVA as an adhesion molecule, but also the pathophysiology of this disease.

\section{Acknowledgments}

We thank Dr. Grant Anhalt (Johns Hopkins University) for advice on the neonatal mouse model of PV and for PV sera, Drs. Takashi Hashi- moto and Takeji Nishikawa (Keio University) for PV sera, and Drs. Stephen Katz and Mark Udey (Dermatology Branch, National Institutes of Health) for reviewing the manuscript.

\section{References}

1. Stanley, J. R. 1989. Pemphigus and pemphigoid as paradigms of organ-specific, autoantibody-mediated diseases. J. Clin. Invest. 83:1443-1448.

2. Stanley, J. R., L. Koulu, and C. Thivolet. 1984. Distinction between epidermal antigens binding pemphigus vulgaris and pemphigus foliaceus autoantibodies. J. Clin. Invest. 74:313-320.

3. Eyre, R. W., and J. R. Stanley. 1988. Identification of pemphigus vulgaris antigen extracted from normal human epidermis and comparison with pemphigus foliaceus antigen. J. Clin. Invest. 81:807-812.

4. Jones, J. C. R., K. M. Yokoo, and R. D. Goldman. 1986. Further analysis of pemphigus autoantibodies and their use in studies on the heterogeneity, structure, and function of desmosomes. J. Cell Biol. 102:1109-1117.

5. Hashimoto, T., M. M. Ogawa, A. Konohana, and T. Nishikawa. 1990. Detection of pemphigus vulgaris and pemphigus foliaceus antigens by immunoblot analysis using different antigen sources. J. Invest. Dermatol. 94:327-331.

6. Amagai, M., V. Klaus-Kovtun, and J. R. Stanley. 1991. Autoantibodies against a novel epithelial cadherin in pemphigus vulgaris, a disease of cell adhesion. Cell. 67:869-877.

7. Takeichi, M. 1991. Cadherin cell adhesion receptors as a morphogenetic regulator. Science (Wash. DC). 251:1451-1455.

8. Takeichi, M. 1990. Cadherins: a molecular family important in selective cell-cell adhesion. Annu. Rev. Biochem. 59:237-252.

9. Ringwald, M., R. Schuh, D. Vestweber, H. Eistetter, F. Lottspeich, J. Engel, R. Dolz, F. Jahnig, J. Epplen, S. Mayer, et al. 1987. The structure of cell adhesion molecule uvomorulin: insights into the molecular mechanism of $\mathrm{Ca} 2+$-dependent cell adhesion. EMBO (Eur. Mol. Biol. Organ.) J. 6:3647-3653.

10. Nagafuchi, A., Y. Shirayoshi, K. Okazaki, K. Yasuda, and M. Takeichi 1987. Transformation of cell adhesion properties by exogenously introduced $\mathrm{E}$ cadherin cDNA. Nature (Lond.). 329:341-343.

11. Hatta, K., A. Nose, A. Nagafuchi, and M. Takeichi. 1988. Cloning and expression of cDNA encoding a neural calcium-dependent cell adhesion molecule: its identity in the cadherin gene family. J. Cell Biol. 106:873-881.

12. Miyatani, S., K. Shimamura, M. Hatta, A. Nagafuchi, A. Nose, M. Matsunaga, K. Hatta, and M. Takeichi. 1989. Neural cadherin: role in selective cellcell adhesion. Science (Wash. DC). 245:631-635.

13. Walsh, F. S., C. H. Barton, W. Putt, S. E. Moore, D. Kesell, N. Spurr, and P. N. Goodfellow. 1990. The N-cadherin gene maps to human chromosome 18 and is not linked to the E-cadherin gene. J. Neurochem. 55:805-812.

14. Nose, A., A. Nagafuchi, and M. Takeichi. 1987. Isolation of placental cadherin cDNA: identification of a novel gene family of cell-cell adhesion molecules. EMBO (Eur. Mol. Biol. Organ.) J. 6:3655-3661.

15. Shimoyama, Y., T. Yoshida, M. Terada, Y. Shimosato, O. Abe, and S. Hirohashi. 1989. Molecular cloning of a human $\mathrm{Ca}^{2+}$-dependent cell-cell adhesion molecule homologous to mouse placental cadherin: its low expression in human placental tissues. J. Cell Biol. 109:1787-1794.

16. Gallin, W. J., B. C. Sorkin, G. M. Edelman, and B. A. Cunningham. 1987. Sequence analysis of a cDNA clone encoding the liver cell adhesion molecule, L-CAM. Proc. Natl. Acad. Sci. USA. 84:2808-2812.

17. Magee, A. I., and R. S. Buxton. 1991. Transmembrane molecular assemblies regulated by the greater cadherin family. Curr. Opin. Cell Biol. 3:854-861.

18. Koch, P. J., M. J. Walsh, M. Schmelz, M. D. Goldschmidt, R. Zimbelmann, and W. W. Franke. 1990. Identification of desmoglein, a constitutive desmosomal glycoprotein, as a member of the cadherin family of cell adhesion molecules. Eur. J. Cell Biol. 53:1-12.

19. Goodwin, L., J. E. Hill, K. Raynor, L. Raszi, M. Manabe, and P. Cowin. 1990. Desmoglein shows extensive homology to the cadherin family of cell adhesion molecules. Biochem. Biophys. Res. Commun. 173:1224-1230.

20. Wheeler, G. N., A. E. Parker, C. L. Thomas, P. Ataliotis, D. Poynter, J. Arnemann, A. J. Rutman, S. C. Pidsley, F. M. Watt, D. A. Rees, et al. 1991. Desmosumal glycoprotein DGI, a component of intercellular desmosome junctions, is related to the cadherin family of cell adhesion molecules. Proc. Natl. Acad. Sci. USA. 88:4796-4800.

21. Nilles, L. A., D. A. D. Parry, E. E. Powers, B. D. Angst, R. M. Wagner, and K. J. Green. 1991. Structural analysis and expression of human desmoglein: a cadherin-like component of the desmosome. J. Cell Sci. 99:809-821.

22. Collins, J. E., P. K. Legan, T. P. Kenny, J. MacGarvie, J. L. Holton, and D. R. Garrod. 1991. Cloning and sequence analysis of desmosomal glycoproteins 2 and 3 (desmocollins): cadherin-like desmosomal adhesion molecules with heterogeneous cytoplasmic domains. J. Cell Biol. 113:381-391.

23. Mechanic, S., K. Raynor, J. E. Hill, and P. Cowin. 1991. Desmocollins form a distinct subset of the cadherin family of cell adhesion molecules. Proc. Natl. Acad. Sci. USA. 88:4476-4480. 
24. Nose, A., K. Tsuji, and M. Takeichi. 1990. Localization of specificity determining sites in cadherin cell adhesion molecules. Cell. 61:147-155.

25. Blaschuk, O. W., R. Sullivan, S. David, and Y. Pouliot. 1990. Identification of a cadherin cell adhesion recognition sequence. Dev. Biol. 139:227-229.

26. Koulu, L., A. Kusumi, M. S. Steinberg, V. Klaus Kovtun, and J. R. Stanley. 1984. Human autoantibodies against a desmosomal core protein in pemphigus foliaceus. J. Exp. Med. 160:1509-1518.

27. Stanley, J. R., L. Koulu, V. Klaus Kovtun, and M. S. Steinberg. 1986. A monoclonal antibody to the desmosomal glycoprotein desmoglein I binds the same polypeptide as human autoantibodies in pemphigus foliaceus. J. Immunol. 136:1227-1230.

28. Eyre, R. W., and J. R. Stanley. 1987. Human autoantibodies against a desmosomal protein complex with a calcium-sensitive epitope are characteristic of pemphigus foliaceus patients. J. Exp. Med. 165:1719-1724.

29. Stanley, J. R., O. M. Alvarez, E. W. Jr.-Bere, W. H. Eaglstein, and S. I. Katz. 1981. Detection of basement membrane zone antigens during epidermal wound healing in pigs. J. Invest. Dermatol. 77:240-243.

30. Towbin, H., T. Staehelin, and J. Gordon. 1979. Electrophoretic transfer of proteins from polyacrylamide gels to nitrocellulose sheets: procedure and some applications. Proc. Natl. Acad. Sci. USA. 76:4350-4354.

31. Anhalt, G. J., R. S. Labib, J. J. Voorhees, T. F. Beals, and L. A. Diaz. 1982. Induction of pemphigus in neonatal mice by passive transfer of $\mathrm{IgG}$ from patients with the disease. N. Engl. J. Med. 306:1189-1196.

32. Takahashi, Y., H. P. Patel, R. S. Labib, L. A. Diaz, and G. J. Anhalt. 1985. Experimentally induced pemphigus vulgaris in neonatal BALB/c mice: a timecourse study of clinical, immunologic, ultrastructural, and cytochemical changes. J. Invest. Dermatol. 84:41-46.

33. Hashimoto, K., K. M. Shafran, P. S. Webber, G. S. Lazarus, and K. H. Singer. 1983. Anti-cell surface pemphigus autoantibody stimulates plasminogen activator activity of human epidermal cells. J. Exp. Med. 157:259-272.

34. Nishikawa, T., S. Kurihara, T. Harada, M. Sugawara, and H. Hatano. 1977. Capability of complement fixation of pemphigus antibodies in vitro. Arch Dermatol. Res. 260:1-6.

35. Jordon, R. E., and L. L. Bushkell. 1979. The complement system in pemphigus, bullous pemphigoid and herpes gestationis. Int. J. Dermatol. 18:271281. 